\title{
Der Einsatz des Zweifels bei Montaigne
}

Montaigne gilt in der Regel als Vertreter einer spezifisch neuzeitlichen Skepsis. Ohne Frage hat die Lektüre von Sextus Empiricus in seinen Essais gewaltige Spuren hinterlassen und ebenso unübersehbar ist die Abneigung, die Montaigne gegenüber dogmatischen Setzungen hegt. In einem Kontrast dazu stehen jedoch viele Stellen, an denen sich Montaigne leichtgläubig zeigt. Er scheint seine Überlegungen fast auf jeder Seite auf irgendwelche gehörten oder meist gelesenen Geschichten zu stützen, deren Glaubwürdigkeit häufig nicht allzu hoch einzuschätzen ist und die sich gerade wegen ihrer offensichtlichen Unwahrscheinlichkeit mit so grossem Interesse erzählen lassen. Der Eindruck der Leichtgläubigkeit wird noch verstärkt durch die Fraglosigkeit, mit der Montaigne seinen katholischen Glauben bekennt, dies zu einer Zeit, in der in Religionsfragen alles in Bewegung ist. Diese Spannung zwischen Skepsis und Leichtgläubigkeit löst sich auf, sobald man die spezifische Rolle des Zweifels bei Montaigne näher betrachtet, wie das in der jüngeren Forschung der Fall ist.

Doch zunächst eine Bemerkung zum Charakter seines Werkes, den Essais: Montaigne hat an diesem Werk in den letzten zwei Jahrzehnten seines Lebens geschrieben, von 1572 bis 1592, seinem Todesjahr. ${ }^{1}$ Das Werk hat in seinem Entstehungsprozess seine Natur stark verändert. Montaigne hatte $\mathrm{zu}$ schreiben begonnen, um sich bei seinen mannigfaltigen Lektüren nicht völlig $\mathrm{zu}$ verlieren. Das

\footnotetext{
1 Die erste Ausgabe von 1580 umfasste zwei Bücher, die zweite von 1588 deren drei. 1595 erscheint eine postume Ausgabe, besorgt von Marie de Gournay. Da Montaigne mit zwei Exemplaren seines Werkes gearbeitet hatte und in beide handschriftliche Zusätze eingefügt hatte, gibt es indessen noch eine weitere Ausgabe, die Ausgabe gemäss dem rexemplaire de Bordeaux`. Die Ausgabe von Villey/Saulnier, nach der Montaigne meistens zitiert wird, folgt diesem sexemplaire de Bordeaux،. Die französischen Zitate mit Seitenangaben in Klammern beziehen sich auf diese Ausgabe: P.Villey/V.-L. Saulnier/M.Conche, Hg., Michel de Montaigne: Les essais. Nouvelle édition, Quadrige. Grands textes, Paris, 2004. Dieser Text ist seitengleich auch online verfügbar unter: http://www.lib.uchicago.edu/efts/ARTFL/projects/montaigne/. Ich zitiere den Originaltext nach dieser Ausgabe in Klammern, die deutsche Übersetzung nach der Übersetzung von Hans Stilett, Michel de Montaigne, Essais. Erste moderne Gesamtübersetzung, Frankfurt am Main, 1998, jeweils mit Spaltenangaben. - Ich verdanke der kritischen Lektüre einer ersten Fassung dieses Textes durch Elmar Holenstein, Andreas Hunziker, Erwin Sonderegger und Josy Zürcher viele konstruktive Vorschläge.
} 
Werk wurde zunehmend zu einem Spiegel seiner selbst bei diesen Lektüren, allerdings zu einem Spiegel, der ihn nicht wiedergibt, wie er ist, sondern der ihn formt, so dass er schreiben kann:

"Je n'ay pas plus faict mon livre que mon livre m'a faict, livre consubstantiel à son autheur, d'une occupation propre, membre de ma vie ; non d'une occupation et fin tierce et estrangere comme tous autres livres." $(665)^{2}$

Eine Spezialität seines Schreibens ist, dass er in der zweiten und der vorbereiteten dritten Version grundsätzlich nie etwas korrigiert, das er früher geschrieben hat. ${ }^{3}$ Denn er will sich in seiner ganzen Widersprüchlichkeit präsentieren. Die Gegensätze und das Schwanken des Urteils liegen in seiner Natur, sie gehören daher zu deren Darstellung. Sein Buch ist ein Gemälde seiner selbst, das er fortlaufend erweitert. Sein Buch handelt von ihm und seinem Schreiben und ist daher selbstbezüglich. ${ }^{4}$ Der besondere Charakter dieses Buches lässt nicht erwarten, dass Montaigne eine bestimmte Position vertritt. Er schreibt von seinem Buch: "Il est plus riche que moy, si je suis plus sage que luy.« $(402)^{5}$ Das Buch weiss mehr, auch mehr über Montaigne, als Montaigne selbst. Und so kann es durchaus Sinn ergeben, in seinem Buch nach der Haltung zu einer bestimmten Frage, wie etwa der Skepsis, zu suchen, auch wenn man sich dabei bewusst zu sein hat, dass Montaigne kein letztes Wort zu einer bestimmten Frage geschrieben hat, sondern eben immer weiterschreibend auf neue Einsichten stiess, was man selbst schon als Beleg für eine Haltung im Stil der pyrrhonischen Skepsis nehmen könnte. Doch der Stil, in dem er schreibt und der Charakter seines Buches unterscheiden sich massiv von demjenigen von Sextus Empiricus.

Es wäre daher überraschend, wenn eine einfache Zuschreibung wie die, dass Montaigne ein pyrrhonischer Skeptiker sei, zutreffen würde. Es ist fraglos, dass er Pyrrho positiv hervorhebt und die Schriften von Sextus Empiricus ganz offensichtlich gut kennt. Doch sein Kontext ist ein anderer als derjenige von Pyrrho oder von Sextus Empiricus. Montaigne schreibt in einem Frankreich, das

2 "Ich habe mein Buch nicht mehr gemacht, als es mich gemacht hat: ein Buch, das mit seinem Autor wesensgleich ist, nur mit mir beschäftigt, unabdingbarer Teil meines Lebens und nicht auf außerhalb seiner selbst liegende Ziele gerichtet wie alle Bücher sonst." (330/1.)

3 963; 484/1.

4 1069; 539/r.

5 "Es ist reicher als ich, selbst wenn ich weiser sein sollte." $(199 / \mathrm{r})$. 
von den Konfessionskriegen erschüttert wird. Der Konflikt um die richtige Konfession wird erbittert ausgetragen, und er geht mitten durch Familien, auch diejenige von Montaigne. Der Blick in die Geistesgeschichte, insbesondere der Antike, lehrt Montaigne, dass menschliche Erkenntnis in Religionsdingen nie diese Gewissheit haben kann, welche diese Auseinandersetzungen rechtfertigen könnte. Es ist gerade die Frage der Erkenntnis Gottes und des göttlichen Willens, der Montaigne am konsequentesten mit Skepsis begegnet.

\section{Formen der Skepsis - Pyrrho und Pyrrhonismus}

Montaigne interessiert sich mehr für Personen und ihre Geschichten als für theoretische Konzepte, daher wird Pyrrho als Gestalt, um den sich Geschichten ranken, an verschiedenen Stellen erwähnt. Marcel Conche und Robert Aulotte unterscheiden zwischen dem Pyrrhonismus von Pyrrho und dem Pyrrhonismus von Sextus Empiricus $^{6}$ : Sextus Empiricus stellt den Pyrrhonismus als eine Philosophie dar, welche Erscheinungen und damit verbundene Urteile mit andern Erscheinungen und den damit verbundenen Urteilen neutralisiert und dadurch zu einer Suspension des Urteils gelangt. Die Erscheinungen sind nach dieser Betrachtungsweise alles, was wir haben. Das heisst jedoch nicht, dass es nicht etwas jenseits der Phänomene gibt, nur bleibt uns dies prinzipiell verborgen. Was wir von Pyrrho anekdotisch wissen, veranlasst Conche und Aulotte, ihm die noch radikalere Position zuzuschreiben, dass er die Idee des Seins im Denken über die Dinge ganz suspendiert habe. Die Unterscheidung zwischen Sein und Erscheinung wird ganz hinfällig, von einer Sache kann man nicht sagen, sie sei oder sie sei nicht. Alles ist Erscheinung - und damit natürlich auch wieder nicht bloss Erscheinung. Montaigne unterscheidet nicht zwischen diesen zwei Sichtweisen.

"Tantôt Montaigne insiste sur le doute enquêteur, sur la nécessaire surseance du jugement, suivant ainsi le scepticisme phénoméniste. Tantôt, comme les pyrrhoniens, il parle, non plus simplement de suspendre son jugement, mais de s'en abstenir, dans une sorte de repos complet. $\ll^{7}$

\footnotetext{
${ }_{6}$ Allerdings soll das hier nicht als philosophiegeschichtliche These vertreten werden, dafür ist die Basis dessen, was wir über Pyrrho wissen, zu schmal. Einige Aussagen, die von Diogenes Laertios berichtet werden, lassen aber eine solche Unterscheidung zu. 7 R.Aulotte, Montaigne: Apologie de Raimond Sebond. Paris 1979, 89; er referiert die Auffassung von Marcel Conche.
} 
Anders als zu Pyrrho, von dem nichts Schriftliches erhalten ist, haben wir von Sextus Empiricus Schriften, die zur Zeit von Montaigne ins Französische übersetzt worden waren und grossen Einfluss gewannen. Markus Wild, der dafür plädiert, Montaigne als pyrrhonischen Skeptiker zu lesen, weist die beiden zentralen Elemente der pyrrhonischen Skepsis gemäss Sextus Empiricus, die Methode der Entgegensetzung von sich widersprechenden Aussagen und der Hinweis auf die Subjektivität der Urteile, bei Montaigne nach. Dabei belässt er es jedoch nicht mit dem Aufweis dieses von ihm genannten Minimalkonsenses, sondern stützt sein Urteil auf eine verfeinerte Charakteristik und es gelingt ihm zu zeigen, dass sich bei Montaigne alle wesentlichen Elemente der Skepsis gemäss Sextus Empiricus ausmachen lassen. ${ }^{8}$

Doch gleichzeitig gibt es etliche Hinweise, die zweifeln lassen, ob sich Montaigne selber als Skeptiker sah. So spricht er despektierlich von ihren Refrains, den standardisierten Antworten, welche darauf abzielen, sich der Anschuldigung des Dogmatismus zu entziehen:

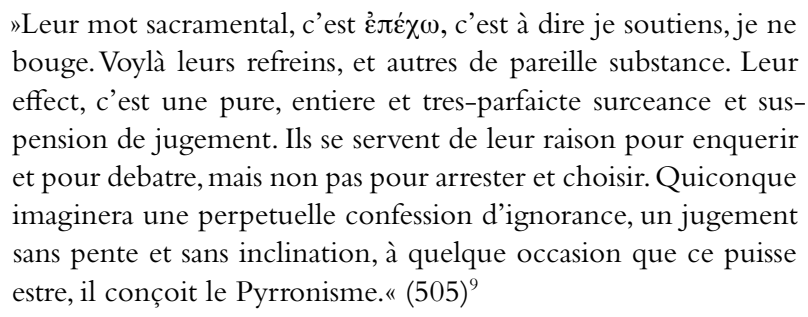

Montaignes Essais leben nicht von solchen Kehrreimen. Die fast krankhafte Bemühung, möglichst jede Äusserung mit ihrem Gegenteil zu neutralisieren, die findet man bei Montaigne nicht, aber man findet, dass er sich auf eine gleichsam natürliche Weise ständig widerspricht, dass ihm Beispiele einfallen, welche das eben Geschriebene wieder relativieren. Das gehört allerdings weniger

8 M. Wild, Montaigne als pyrrhonischer Skeptiker. In: C. Spoerhase/D.Werle/M. Wild (Hg.), Unsicheres Wissen. Skeptizismus und Wahrscheinlichkeit 1550-1850. Berlin/New York, 2009.

9 "Ihr sakrosanktes Wort lautet દ̇đé $\chi \omega$, das heißt: >Ich enthalte mich, ich lasse mich zu keinem Urteil bewegen. Inhalts. Sie laufen auf eine reine, uneingeschränkte und vollendete Urteilsenthaltung hinaus. So bedienen sich die Pyrrhonisten ihres Verstands denn auch nur zum Forschen und Diskutieren, nicht aber dazu, eine Wahl zu treffen und sich zu entscheiden. Man stelle sich ein fortwährendes Bekennen von Unwissenheit vor, ein völlig unparteiisches, unter keinen Umständen irgendeiner Seite zuneigendes Denken, und man hat vom Pyrrhonismus den richtigen Begriff." (250/r-251/l) 
zu seinem Skeptizismus als vielmehr zu seinem Programm der Selbstbeobachtung. Die Widersprüchlichkeit oder Vielgestaltigkeit ist seine eigene Form.

\section{Erschliessende Skepsis}

Hugo Friedrich hat die Skepsis von Montaigne als erschliessende Skepsis charakterisiert."Seine Skepsis lähmt und verbaut nicht, sondern erschließt. ${ }^{10}$ Er ist auch der Auffassung, dass sich die Skepsis Montaignes in erster Linie an Sextus Empiricus orientiert, hält aber fest: "Aber ganz anders als dort kommt in den Essais diese Beobachtung zur Geltung; sie breitet den komplexen Lebensstoff um seiner selbst willen aus, nicht bloß zur Demonstration eines Schulprinzips. ${ }^{11}$ Diese Auffassung hat durch Marc Foglia in jüngerer Zeit eine Vertiefung erfahren. ${ }^{12}$ Er geht in seinem online zugänglichen Artikel von der Fragestellung aus, ob man Montaigne nicht ganz anders denn als Skeptiker verstehen könnte. Und er stellt fest: "Contrairement à ce soutient l'interprétation sceptique, les Essais ne sont pas orientés vers l'arrêt du jugement, mais vers l'activité du jugement lui-même. $\aleph^{13}$ Tatsächlich ist das Üben oder gar Trainieren der Urteilskraft ein wichtiges Anliegen Montaignes. Er betont an mehreren Stellen die Schwäche seines Gedächtnisses. Doch dies wird seines Erachtens durch das ständige Üben der Urteilskraft kompensiert. Die Essais sind die Erprobung seiner Urteilskraft. Montaigne nimmt sich fast beliebige Gegenstände vor, nur um an ihnen seine Urteilskraft zu erproben. ${ }^{14}$

»Tant y a que je me contredits bien à l'adventure, mais la verité, comme disoit Demades, je ne la contredy point. Si mon ame pouvoit prendre pied, je ne m'essaierois pas, je me resoudrois : elle est tousjours en apprentissage et en espreuve.« $(805)^{15}$

\footnotetext{
${ }^{10}$ H. Friedrich, Montaigne, 2., neubearb. Aufl. Bern, 1967, 125.

${ }^{11}$ Ebd.

${ }^{12}$ M. Foglia, La place du scepticisme dans la philosophie de Montaigne, 2005, http:// www.e-litterature.net/publier2/spip/spip.php?page $=$ article5\&id_article $=197$

${ }_{13}^{13}$ Ebd.

${ }^{14}$ Conche, zit. in Foglia, 2005.

15 „Daher mag ich mir zwar zuweilen widersprechen, aber der Wahrheit, wie Demades sagte, widerspreche ich nie. Könnte meine Seele jemals Fuß fassen, würde ich nichtVersuche mit mir machen, sondern mich entscheiden. Doch sie ist ständig in der Lehre und Erprobung." (398/r-399/1)
} 
Es geht Montaigne um das Ausloten dessen, was der Mensch ist, an seinem eigenen Beispiel. Das, was als Widerspruch erscheint, erweitert den Horizont der menschlichen Möglichkeiten. An der eben zitierten Stelle fährt er fort:

"Je propose une vie basse et sans lustre, c'est tout un. On attache aussi bien toute la philosophie morale à une vie populaire et privée que à une vie de plus riche estoffe: chaque homme porte la forme entiere de l'humaine condition. ${ }^{16}$

Montaigne lässt an verschiedenen Stellen durchblicken, dass er durchaus der Auffassung ist, seine Essais würden etwas von dieser hhumaine condition erschliessen. An gewissen Stellen wird gar deutlich, dass das Schwanken des Urteils weniger mit der Unsicherheit unserer Erkenntnis zu tun hat, als vielmehr mit dem objektiven Schwanken der Dinge selbst. "Certes, c'est un subject merveilleusement vain, divers, et ondoyant, que l'homme. Il est malaisé d'y fonder jugement constant et uniforme." (9) ${ }^{17}$ Aber das, was erschlossen wird, gehört in den Bereich der Weisheit, nicht des Wissens. Der Weise, das Ideal der hellenistischen Philosophien, in deren Umkreis sich Montaigne lesend aufhält, strebt nach einer sich selbst genügenden Lebensform. Sein Wissen besteht in Erfahrung, nicht in einer Anhäufung von Kenntnissen. Er sagt von sich, er wolle weiser werden, nicht gelehrter $(414 ; 206 / r)$. Nicht, dass Montaigne behaupten würde, er sei tatsächlich weise. ${ }^{18}$ Seine Weisheit liegt gerade im richtigen Genuss des Lebens und nicht auf ein bestimmtes Ziel hin zu leben. "Mon mestier et mon art, c'est vivre." $(379 ; 188 / 1)$ "Ce ne sont mes gestes que j'escris, c'est moy, c'est mon essence.« $(379 ; 188 / 1)^{19}$

Die Prüfung der Urteile und Vorurteile hilft Montaigne, die schwankende, vielseitige und auch widersprüchliche menschliche Lebensart von allen Seiten zu betrachten, nach Möglichkeit, ohne

16 »Ich führe ein Leben ohne Glanz und Gloria vor Augen - warum auch nicht? Man kann alle Moralphilosophie ebensogut auf ein niedriges und namenloses wie auf ein reicher ausgestattetes Leben gründen: Jeder Mensch trägt die ganze Gestalt des Menschseins in sich."(399/1)

17 "Wahrlich der Mensch ist ein seltsam wahnhaftes, widersprüchliches, hin und her schwankendes Wesen! Es fällt schwer, ein gleichbleibendes und einheitliches Urteil darauf zu gründen. « $(10 / \mathrm{r})$

${ }^{18}$ Und er weiss auch: "Le plus sage homme qui fut onques, quand on luy demanda ce qu'il sçavoit, respondit qu'il sçavoit cela, qu'il ne sçavoit rien.« (501) / "Sokrates, der weiseste Mann, den es je gab, pflegte auf die Frage, was er wisse, zu antworten: Er wisse, daß er nichts wisse." $(248 / \mathrm{r})$

19 "Mein Handwerk und meine Kunst ist es, zu leben." (188/1); NNicht einzelne Akte beschreibe ich daher, sondern mich, sondern mein ganzes Wesen. " $(188 / 1)$ 
dabei mit wertenden Urteilen Zensur zu üben. Die Lektüre der Essais vermittelt weniger den Eindruck, dass wir letztendlich nichts wissen als den des Reichtums der menschlichen Existenz. Man lese beliebige fünf Seiten bei Sextus Empiricus und bei Montaigne und man wird ohne weiteres einen völlig andern Atem feststellen, auch eine andere Lebenslust, ein anderes Interesse am Menschlichen, das ihm nie Allzumenschliches ist.

Das Buch von Montaigne lässt sich auf keine einfache Formel bringen. Die Erkundung seiner selbst im Spiegel aller möglichen Geschichten, wahrscheinlichen und ganz unwahrscheinlichen gleichermassen, ist nicht auf ein Resultat angelegt, sondern auf ein immer reichhaltigeres Gemälde. ${ }^{20}$ Seine Vorgehensweise schildert er so:

"Je prends de la fortune le premier argument. Ils me sont également bons. Et ne desseigne jamais de les produire entiers. Car je ne voy le tout de rien: Ne font pas, ceux qui promettent de nous le faire veoir. De cent membres et visages qu'a chaque chose, j'en prens un tantost à lecher seulement, tantost à effleurer; et par fois à pincer jusqu'à l'os. J'y donne une poincte, non pas le plus largement, mais le plus profondement que je sçay. Et aime plus souvent à les saisir par quelque lustre inusité. Je me hazarderoy de traitter à fons quelque matière, si je me connoissoy moins. Semant icy un mot, icy un autre, eschantillons despris de leur piece, escartez, sans dessein et sans promesse, je ne suis pas tenu d'en faire bon, ny de m'y tenir moy mesme, sans varier quand il me plaist; et me rendre au doubte et incertitude, et à ma maistresse forme, qui est l'ignorance. Tout mouvement nous descouvre." $(302)^{21}$

\footnotetext{
${ }^{20}$ Die Metapher des Malens findet sich schon im Hinweis an den Leser am Anfang des Buches. Eine weitere Stelle: 665; 330/1.

21 "Stets ergreife ich die erstbeste Sache, die der Zufall mir bietet. Ich finde sie alle gleich gut. Dabei plane ich nie, sie erschöpfend darzulegen, denn von nichts sehe ich das Ganze (noch tun das jene, die versprechen, es uns sehn zu lassen). Von den hundert Gliedern und Gesichtern, die jedes Ding hat, nehme ich mir jeweils eins vor, zuweilen um bloß daran zu lecken, zuweilen um seine Oberfläche abzutasten; öfters aber auch, um bis zu den Knochen vorzustoßen. Ich möchte nicht so breit, sondern so tief eindringen, wie ich nur kann; und meistens liebe ich es, die Dinge hierbei von einer ungewöhnlichen Seite her in den Griff zu nehmen. Gewiß würde ich es wagen, einen Gegenstand von Grund auf abzuhandeln, wenn ich mich weniger gut kennte und vor meinem Unvermögen die Augen schlösse. So aber lasse ich hier ein Wort und dort ein andres als dem Ganzen zusammenhanglos entnommene Probestückchen fallen, ohne Plan und Versprechen, und bin deshalb weder dem Leser noch mir selbst gegenüber verpflichtet, mich streng an die jeweilige Sache zu halten; vielmehr kann ich nach Lust und Laune meine Meinung ändern und mich dem Zweifel und der
} 


\section{Dialektischer Gang der Selbstprüfung}

Auch wenn Montaigne hier und an andern Stellen betont, dass er ohne Plan und Absicht vorgeht, dass er sich dort aufhält, wo es ihm gefällt, möglichst im Unvorhergesehenen, so heisst das doch nicht, dass sich nicht Muster ergeben, die eine sorgfältige Lektüre aufzudecken weiss. Solche Muster müssen dem Autor nicht gegenwärtig sein, sie können eine Tendenz verraten, worüber der Autor nicht unbedingt volle Klarheit erlangt hat, denn wie schon zitiert: Das Buch weiss mehr als Montaigne selbst. Anne Hartle hat eine Deutung von Montaigne vorgelegt, welche seine Skepsis ebenfalls als erschliessende Skepsis versteht, die sich einem dialektischen Gang entfaltet. ${ }^{22}$ Verborgene Neigungen dieses Schriftstellers werden offengelegt. In ihrem bahnbrechenden Buch ist von Montaigne als einem Skeptiker nicht die Rede, sondern bloss von seiner zirkulären Dialektik. ${ }^{23}$ Ich stütze mich im Folgenden auf den Aufsatz, verweise für eine Vertiefung aber auf das Buch.

Hartle fasst die skeptischen Argumente in der Apologie de Raimond Sebond auf konzise Weise zusammen. ${ }^{24}$ Sie stellt dann aber fest:

"Most of those who regard Montaigne as a skeptic do, however, acknowledge that there are decidedly non-skeptical features of his thought which are at least difficult to reconcile with a thoroughgoing self-conscious skepticism. ${ }^{25}$

Hartle ist daran gelegen, die hier eingangs erwähnte Spannung zwischen Skepsis und Leichtgläubigkeit aufzulösen. Dies gelingt, indem sie Montaigne letztlich nicht als einen Skeptiker, sondern als einen Sokratiker versteht. Wenn man bedenkt, dass das Zentrum der Essais in der Selbsterprobung, der Selbstprüfung liegt, ist das plausibel.

Hartle identifiziert bei Montaigne einen dialektischen Dreischritt. Dieser geht vom gewöhnlichen Menschen ${ }^{26}$, bzw. von den Einstellungen des gewöhnlichen Menschen aus und führt über die Reflexion dieser Position zu einer belehrten Einsicht darin, dass in diesem scheinbar einfachen Standpunkt eine Weisheit liegt, die zunächst nicht erkannt wird. Der scheinbare Gegensatz von

Ungewisheit anheimgeben - sowie meinem maßgeblichen Wesenszug: dem Nichtwissen. Was immer wir tun, enthüllt uns."(153/1)

${ }^{22}$ A. Hartle, Montaigne and skepticism. In: Ullrich Langer (Hg.): The Cambridge companion to Montaigne. Cambridge, 2005,183-206.

23 A. Hartle, Michel de Montaigne. Accidental Philosopher. Cambridge 2007.

${ }^{24}$ Hartle, 2005, 186-189.

${ }^{25}$ Ebd., 189

26 ,Gewöhnlich im Sinne des oben zitierten "vie populaire et privée». 
Skeptizismus und Leichtgläubigkeit löst sich dabei auf. Denn auch noch die unwahrscheinlichsten Geschichten sind Beispiele der menschlichen Einbildungskraft und d.h. der Möglichkeit, sich Dinge, Ereignisse und Lebensmöglichkeiten vorzustellen. Der Skeptiker in diesem Sinne ist derjenige, der das Mögliche eben möglichst von der Bindung an das scheinbar einzig Wirkliche befreit. Montaigne erzählt viele Geschichtchen, dabei ganz unglaubwürdige, doch dessen ist er sich bewusst:

"Aussi en l'estude que je traitte de noz moeurs et mouvemens, les tesmoignages fabuleux, pourveu qu'ils soient possibles, y servent comme les vrais. Advenu ou non advenu, à Paris ou à Rome, à Jean ou à Pierre, c'est toujours un tour de l'humaine capacité, duquel je suis utilement advisé par ce recit.« $(105)^{27}$

Die Geschichtchen müssen also gar nicht wahr sein, sie dienen einzig dazu, den Bereich des Möglichen und des Glaubbaren auszuloten. Skepsis und Leichtgläubigkeit bedingen sich hier: Das genaue Hinsehen soll nicht durch Vorurteile über das, was als möglich gilt, verhindert werden, auch dem Unwahrscheinlichen wird eine Chance gegeben und der Raum des Denkbaren damit ausgeweitet, ohne dass man deswegen allem auch Glauben schenkt. »Montaigne's skepticism, then, is not the doubt of the ancient skeptics but rather an openness to what is possible and an overcoming of presumption at the deepest level. ${ }^{28}$ Tatsächlich geht es nicht um einen Zweifel, der Gewissheiten aushebelt, sondern der das unbefragt Gegebene in ein neues Licht rückt und neue Dimensionen des Verstehens von ganz Alltäglichem eröffnet, indem seine Selbstverständlichkeit reduziert wird.

"The movement of Montaigne's thought is first to open us to the possibility of the strange and foreign, then lead us back to the familiar and let us see the extraordinary in the ordinary, in the familiar and the common. $\mathbb{1}^{29}$

Diese Denkbewegung ist auch relevant, wenn es um das Verständnis von Montaignes Konservativismus geht. Dieser bezieht sich auf po-

\footnotetext{
${ }^{27}$ „Bei meinen Untersuchungen unserer Beweggründe und Verhaltensweisen sind mir jedenfalls die erdichteten Zeugnisse, soweit sie möglich scheinen, ebenso dienlich wie die wahren. Geschehen oder nicht, in Paris oder Rom, dem Hinz oder Kunz stets zeigen sie mir, wozu Menschen fähig sind, und das zu wissen ist mir nützlich." (59/1)

${ }^{28}$ Hartle, 2005, 193.

${ }^{29}$ Ebd., 194.
} 
litische wie auch auf religiöse Verhältnisse. Montaigne plädiert für ein Festhalten am Vorhandenen. Das heisst aber nicht, dass er dieses nicht zur Bewährung ausgesetzt sehen würde. Wenn es etwa um den Gegensatz von Selbstregierung und Monarchie geht, sieht er nicht per se in einem der beiden Systeme einen Vorteil. Was es zu vermeiden gilt, sind die Auseinandersetzungen um das richtige System. Es ist die Auffassung, dass eines der beiden das richtige sei, das zu Konflikten führt, von denen letztendlich niemand profitiert, auch nicht die sogenannte Wahrheit. Denn die Wahrheit entzieht sich uns ja gerade. Der Dreischritt von Hartle ist hier leicht einsehbar: Zunächst gibt es den Hang, am Bekannten festzuhalten. Dann folgt der Zweifel, dass das eigene Bekannte ja für viele andere einfach das Fremde und gar nicht Naheliegende ist. Darauf folgt die Einsicht, dass dieses Bekannte weniger mit Notwendigkeit einhergeht, als man ursprünglich glauben mochte. Das ist kein Grund, es preiszugeben. Dies führt vielmehr zu einer etwas distanzierten Haltung gegenüber scheinbar so zentralen Fragen wie der politischen Ordnung und der Religion. Wenn die Einsicht sich einstellt, dass es in diesen Fragen wichtig ist, irgendeine Ordnung zu haben und dagegen ganz sekundär welche, so ist damit hinreichend begründet, am Bestehenden festzuhalten. "Nous sommes Chrestiens à mesme titre que nous sommes ou Perigordins ou Alemans." (445) ${ }^{30}$ Das heisst auch, dass man das eigene Christsein so wenig in Frage stellt wie die Herkunft.

\section{Montaigne und seine Verteidigung der natürlichen Theologie (Sebond)}

Montaigne hält an der Monarchie und am Katholizismus ganz unzweideutig fest, aber nicht, weil er der Auffassung wäre, nur in ihnen sei Heil zu erlangen. Im Folgenden soll seine Haltung zum Katholizismus und zum Christentum näher betrachtet werden. Tatsächlich sind sich die Interpreten in dieser Hinsicht ähnlich uneins wie in der Einschätzung seines Skeptizismus. Jean Guiton ${ }^{31}$ unterscheidet vier Gruppen von Einschätzungen: a) Montaigne gibt ein Lippenbekenntnis zum Katholizismus, im übrigen aber zeigt er durch seine durchgängig heidnisch geprägte Moral, dass er nicht gläubig ist (so die Reaktion von Pascal); b) Montaigne spielt eine Komödie, die

\footnotetext{
${ }^{30}$ "Christen sind wir im gleichen Sinne, wie wir Périgorden oder Deutsche sind.» $(220 / \mathrm{r})$

31 Berichtet in Aulotte, 1979, 102ff
} 
ständigen Glaubensbekenntnisse und Akte der Unterwerfung unter die Kirche sind nicht ernst gemeint (Saint-Beuve und dann viele andere, z.B. Léon Brunschvicg und André Gide); c) Montaigne ist ein guter Katholik, jedoch kein guter Christ, indem er zwischen Glaube und Vernunft einen Graben sieht. Es wird ihm vorgeworfen, dass er sich dem Katholizismus unterwirft, aber ohne dabei wirklich religiöse Gefühle zu hegen; d) Montaigne ist ein guter Katholik und infolgedessen auch ein guter Christ, indem er in seiner Apologie, ähnlich wie später Pascal, die Misere des Menschen, der Gott fern ist, aufzeigt. Häufig findet sich auch die Auffassung, Montaigne sei ein Fideist gewesen. ${ }^{32}$

Sind diese Positionen wirklich so gegensätzlich? Auf den ersten Blick bestimmt. Das widersprüchliche Bild ergibt sich daraus, dass vorhandene Facetten des Bildes, das Montaigne von sich malt, etwas gar stark belastet werden. Natürlich gibt es auch Versuche, die verschiedenen Positionen zu vereinbaren, etwa wenn Brahami fragt, ob sich Montaigne als Fideist gibt, um nicht als Freidenker entlarvt $\mathrm{zu}$ werden. ${ }^{33}$ Montaigne geht es in seinem Buch in erster Linie um sich selbst. In seinem Buch schildert er sich nicht als einen, der einen Skeptizismus vertritt, er schildert sich als einen, der viele verschiedene Gedanken hegt, auch über Religion, der sich in vielen verschiedenen Situationen auf eine bestimmte Weise verhält, ohne Anspruch, dies sei die einzig richtige Haltung. Er beobachtet auch, wie er solche Auffassungen rezipiert: Wenn er einen bestimmten Autor liest, neigt er seinen Auffassungen zu, wenn er die Lektüre wechselt, dann wieder einem andern. ${ }^{34}$ Er lässt seinem Denken freien Lauf.

Mit philosophischen und religiösen Strömungen setzt sich Montaigne vor allem in der sog. Apologie de Raimond Sebond auseinander. Dies ist nicht nur das mit Abstand längste Kapitel der Essais, es unterscheidet sich von den andern auch darin, dass hier eben ein bestimmtes Ziel vorgegeben ist: die Verteidigung der Schrift von Sebond. Mit Raimond Sebond hat sich Montaigne zuerst im Auftrag seines Vaters befasst, der ihn bat, die Schrift über die natürliche Theologie des Sebond aus dem Latein ins Französische zu übersetzen.

\footnotetext{
${ }^{32}$ Literatur dazu im Bericht von Richard Amesbury, Artikel "Fideism» in der Stanford Encyclopedia of Philosophy. Vordergründig scheint der Fideismus mit der Position c) identisch zu sein, indem er den Glauben in keiner Weise auf die Vernunft gründet. Doch c) meint ein Lippenbekenntnis zum Katholizismus, insbesondere der Institution, nicht wirklich einen Glauben.

${ }^{33}$ Vgl. H. Stilett, Von der Lust, auf dieser Erde zu leben. Wanderungen durch Montaignes Welten, Franfurt am Main, 2008, 54.

34 Vgl. 875; 438/1.
} 
Die Motivation dazu lag in der um sich greifenden Reformation. Mit der Schrift Theologia naturalis sive liber creaturarum beanspruchte Sebond, die Wahrheit des katholischen Glaubens unfehlbar zu beweisen und jeden andern Glauben als falsch zu widerlegen. Seine Lehre verstand er als voraussetzungslose Grundwissenschaft, die jedermann innerhalb eines Monats ohne Mühe erlernen könne. ${ }^{35}$ Die Erkenntnis Gottes aus der Schöpfung ist der Bibel gleichwertig, da diese aber nur von Klerikern verstanden werde, ist das allen zugängliche Buch der Schöpfung der Bibel vorzuziehen. ${ }^{36}$ Montaigne sieht die Bedeutung dieses Werkes in der Auseinandersetzung mit der Reformation: "ce fut lors que les nouvelletez de Luther commençoient d'entrer en credit et esbranler en beaucoup de lieux nostre ancienne creance.« $(439)^{37}$

Diese Verteidigung von Sebond stellt vor interpretatorische Probleme. Die Schwierigkeiten, Montaignes Denken in Sachen Skeptizismus und Religion einzuschätzen, hängen zu einem nicht geringen Teil an der vertrackten Sonderposition dieses langen Kapitels im Werk von Montaigne. Es ist immer wieder festgestellt worden, dass Montaigne unter dem Vorwand, die Schrift zu verteidigen, sie in ihrem zentralen Anliegen untergrabe und die Glaubensgewissheit, die sich bei Sebond auf die natürliche Theologie stützt, durch einen Skeptizismus ersetze. ${ }^{38}$ Doch schauen wir, wie Montaigne diese Sache angeht. Er beginnt mit einem kräftigen Lob und einer Motivation für seine Verteidigung:

"Je trouvay belles les imaginations de cet autheur, la contexture de son ouvrage bien suyvie, et son dessein plein de pieté. Par ce que beaucoup de gens s'amusent à le lire, et notamment les dames, à qui nous devons plus de service, je me suis trouvé souvent à mesme de les secourir, pour descharger leur livre de deux principales objections qu'on luy faict. Sa fin est hardie et courageuse, car il entreprend, par raisons humaines et naturelles, establir et verifier

35 "Et potest haberi infra medium mensem et sine labore."Raimundus Sabundus, Theologia naturalis sive liber creaturarum, Stuttgart-Bad Cannstatt, 1966, 32^ (Die Seitenzahl mit $\star$ beziehen sich auf den kritisch edierten Prologus).

${ }^{36}$ Ebd., 35*. Sebond vertritt hier die Auffassung, dass das liber creaturarum zugänglicher sei als die Bibel, welche nur über die Interpretation der doctores erschlossen werden könne. Hier sieht er die beiden Bücher klar als Alternativen, wobei sich eben dem Laien das liber creaturarum empfiehlt, aufgrund der Erlernbarkeit in weniger als einem Monat.

37 "[B] egannen damals doch die Neuerungen Luthers Einfluß zu gewinnen und an vielen Orten unsern alten Glauben zu erschüttern.« (217/1)

${ }^{38}$ Wer Montaigne primär als Skeptiker versteht, kommt um die Einschätzung nicht herum, dass er Sebond verteidige, indem er ihn angreift. 
contre les atheistes tous les articles de la religion Chrestienne : en quoy, à dire la verité, je le trouve si ferme et si heureux que je ne pense point qu'il soit possible de mieux faire en cet argument là, et croy que nul ne l'a esgalé.« $(440)^{39}$

Er hat mit seiner Verteidigung zwei Hauptargumente gegenüber dieser Schrift im Blick:

"La premiere reprehension qu'on fait de son ouvrage, c'est que les Chretiens se font tort de vouloir appuyer leur creance par des raisons humaines, qui ne se conçoit que par foy et par une inspiration particuliere de la grace divine." $(440)^{40}$

Dieser erste Einwand wird so formuliert, dass damit eine bestimmte Gruppe von Gläubigen angesprochen wird, die den Glauben allein auf die göttliche Gnade gründen wollen. Es darf vermutet werden, dass Montaigne hier in erster Linie an die Anhänger der Reformation denkt. ${ }^{41}$ Nicht, dass Montaigne das Christentum nicht für reformbedürftig halten würde, doch die Reformation macht die Geschichte nicht besser, indem hier Fragen aufgeworfen werden, welche durch den Menschen so oder so nicht zu entscheiden sind. In seinen Ausführungen zu diesem Argument hält er den deplorablen Zustand des Christentums fest, der weder auf eine durch göttliche Gnade inspirierte Verfassung der Christen, noch auf den tatsächlichen Glauben aufgrund der sichtbaren Schöpfung schliessen lässt. Vielmehr ist der Glaube von Gewohnheiten bestimmt.

\footnotetext{
39 "Ich fand die Gedanken dieses Schriftstellers schön, sein Werk folgerecht gegliedert und seine Absicht voller Frömmigkeit. Da viele Leute es mit großem Interesse lesen, namentlich die Damen, denen wir ja vornehmlich unsre Dienste schulden, bot sich mir schon öfters die Gelegenheit, ihnen bei der Entkräftung der zwei wichtigsten Einwände zu helfen, die man gegen das Buch vorbringt. Das Vorhaben des Autors ist höchst wagemutig, denn er unternimmt es, gegen die Atheisten kraft natürlicher, rein menschlicher Vernunftgründe den Wahrheitsbeweis für alle Glaubenssätze der christlichen Religion zu erbringen, und er tut dies, finde ich, unzweifelhaft mit solcher Sattelfestigkeit und solch glücklicher Hand, daß ich eine überzeugendere Vertretung der Sache für unmöglich halte - niemand ist ihm meines Erachtens hierin je gleichgekommen. « $(217 / \mathrm{r})$

${ }^{40}$ „Der erste Einwand, den man gegen sein Werk vorbringt, besagt, dass die Christen schlecht beraten seien, wenn sie ihre Heilslehre, die nur durch den Glauben und durch eine besondere Eingebung der göttlichen Gnade empfangen werden könne, auf menschliche Vernunftgründe stützen wollten.« (218/1)

${ }^{41}$ Das Werk von Sebond hingegen geht vor die Reformation zurück, es entstand in den Jahren 1434-36.
} 
"Je voy cela evidemment, que nous ne prestons volontiers à la devotion, que les offices qui flattent nos passions. Il n'est point d'hostilité excellente comme la chrestienne. Nostre zele faict merveilles, quand il va secondant nostre pente vers la haine, la cruauté, l'ambition, l'avarice, la detraction, la rebellion. A contrepoil, vers la bonté, la benignité, la temperance, si, comme par miracle, quelque rare complexion ne l'y porte, il ne va ny de pied ny d'aile. Nostre religion est faicte pour extirper les vices ; elle les couvre, les nourrit, les incite." (444) ${ }^{42}$ Montaigne kritisiert hier nicht eine Konfession, sondern das gelebte Christentum, das in keinerlei Übereinstimmung mit den proklamierten Inhalten steht, was sich insbesondere in den Religionskriegen zeigt.

Montaigne scheint auf ein Zusammenwirken von Vernunft und Gnade zu zählen. Zugunsten derVernunft meint er:

"Ce seroit faire tort à la bonté divine, si l'univers ne consentoit à nostre creance. Le ciel, la terre, les elemans, nostre corps et nostre ame, toutes choses y conspirent: il n'est que de trouver le moyen de s'en servir. Elles nous instruisent, si nous sommes capables d'entendre. Car ce monde est un temple tres-sainct, dedans lequel l'homme est introduict pour y contempler des statues, non ouvrées de mortelle main, mais celles que la divine pensée a faict sensibles: le Soleil, les estoilles, les eaux et la terre, pour nous representer les intelligibles." $(447)^{43}$

42 "Ich sehe in aller Klarheit, daß wir nur jene Pflichten der Frömmigkeit bereitwillig erfüllen, mit denen wir zugleich unseren Leidenschaften frönen können. Die Christen übertreffen alle andern an Feindeshaß. Unser Glaubenseifer tut Wunder, wenn er sich mit unsrer Neigung zu Ehrgeiz und Habsucht, zu Verleumdung und Rachgier, zu Grausamkeit und Aufruhr verbündet. Die Gegenrichtung hin zu Mäßigung, Wohlwollen und Güte aber schlägt er, falls ihn nicht wie durch ein Wunder eine höchst seltene Veranlagung hierzu bewegt, weder zu Fuß noch auf Flügeln ein. Unsere Religion ist geschaffen, die Laster auszurotten; doch sie beschirmt sie, zieht sie groß und spornt sie an." $(219 / \mathrm{r})$

43 "Es widerspräche der vollendeten Meisterschaft Gottes, wenn unser Glaube nicht durch das Universum bestätigt würde. Himmel und Erde, die Elemente, unser Körper und unsre Seele - alle Dinge wirken darin zusammen; man braucht nur die Mittel zu finden, uns ihrer zu bedienen. Falls wir aufnahmebereit sind, lernen wir von ihnen, denn diese Welt ist ein hochheiliger Tempel, in den der Mensch zur Betrachtung von Bildsäulen hineingeführt wird: nicht solcher, die sterbliche Hände gestalteten, sondern jener - wie Sonne und Sterne, Gewässer und Erde -, die der göttliche Schöpfergeist unsren Sinnen als Abbilder der unzugänglichen Dinge zugänglich gemacht hat.« $(221 / 1-r)$ 
Mit solchen Überlegungen unterstützt er die Grundaussage von Sebond. Der schwachen Vernunft muss jedoch mit göttlicher Gnade aufgeholfen werden. Das drückt Montaigne an verschiedenen Stellen aus, in diesem Kontext am pointiertesten in der Zusammenfassung zum ersten Einwand: "Or nos raisons et nos discours humains, c'est comme la matiere lourde et sterile : la grace de Dieu en est la forme ; c'est elle qui y donne la façon et le pris." (447) ${ }^{44}$ Montaigne fährt hier fort, dass die tugendhaften Taten von Sokrates und Cato letztlich nutzlos blieben, weil sie Gott noch nicht gekannt hätten. Damit gibt er die Einschätzung von Augustinus in den Confessiones wieder, der den Defekt der antiken Philosophen darin sieht, dass sie Jesus Christus nicht gekannt hätten. ${ }^{45}$ Der Glaube ist für Montaigne auch erforderlich, um die Argumente von Sebond richtig zu verstehen. Diese Argumente machen ihn wiederum für die Gnade Gottes bereit. ${ }^{46}$

Montaigne vermutet am Anfang der Apologie, dieser sonst unbekannte Sebond würde einfach die Argumente von Thomas von Aquin wiedergeben. In seiner Verteidigung von Sebond, die damit rechnet, dass nurVernunft und Gnade gemeinsam ausreichend sein könnten, folgt er sehr genau der Argumentation von Sebond, der schreibt: "Quam quidem sapientiam nullus potest videre nec legere per se in dicto libro semper aperto, nisi sit a Deo illuminatus et a peccato originali mundatus.« (S. 38) ${ }^{47}$ Sebond und auch Montaigne folgen in dieser Auffassung dem Augustinismus des Mittelalters, zu dem Thomas in dieser Hinsicht gerade eine Alternative darstellt.Vernunft und Gnade sind beide auf ein Zusammenspiel angewiesen und stellen nicht einfach zwei unterschiedliche Wege zur einen Wahrheit dar. ${ }^{48}$ Doch in dieser Hinsicht ist Sebond in seinem Prologus nicht sehr konsistent, denn er argumentiert über weite Strecken so, dass man annehmen muss, die Vernunft allein könne sich die Wahrheit der christlichen Religion erschliessen. Allerdings ist dort der Gegenpart

\footnotetext{
44 "Zusammengefasst lässt sich auf den ersten Einwand also erwidern: Unsere menschlichen Überlegungen und Vernunftschlüsse sind gleichsam ungeformter und brachliegender Rohstoff, den in Form zu bringen nur die Gnade Gottes vermag: Sie erst gibt ihm Gestalt und Wert." (221/r)

${ }^{45}$ Augustinus, Confessiones, 3.4.8 und 5.14.25.

46 447; $221 / \mathrm{r}$.

47 „Diese Weisheit kann keiner aus eigenen Stücken im erwähnten immer offenen Buch sehen und verstehen, es sei denn, er sei von Gott erleuchtet und von der Ursünde gereinigt." (meine Übers.)

${ }^{48}$ Vgl. zu Thomas, S.th. II-II, quaest. 8, art. 4, wo sich Thomas zwar auf Augustin bezieht, aber von Gnade gleichwohl in ganz anderer Weise spricht, nämlich als dem Stand, in den sich der Mensch selbst durch seinen Willen versetzt.
} 
nicht die Gnade, sondern die Offenbarung bzw. die nur durch viel hermeneutische Kunst erschliessbare Heilige Schrift.

Die zweite Art von Einwänden gegen Sebond fasst Montaigne so zusammen:

"Aucuns disent que ses argumens sont foibles et ineptes à verifier ce qu'il veut, et entreprennent de les choquer aysément. Il faut secouer ceux cy un peu plus rudement, car ils sont plus dangereux et plus malitieux que les premiers." $(448)^{49}$

Bei dieser zweiten Gruppe handelt es sich, so muss man annehmen, um Rationalisten, welche auf das Element der Gnade ganz verzichten und mit derVernunft allein die Prüfung der Überlegungen von Sebond angehen - und diese für ungenügend befinden. Die richtige Strategie, mit ihnen fertig zu werden, besteht daher darin, die Schwäche der menschlichen Vernunft aufzuzeigen, eine Aufgabe, der sich Montaigne nun mit Gründlichkeit widmet.

Die Skepsis Montaignes untergräbt die Ansprüche dieser Dogmatiker, deren Schwäche darin besteht, ein Wissen zu beanspruchen, das über das Lesen von wahrscheinlichen Zeichen im Sinne einer natürlichen Theologie hinausgeht.

"Que nous presche la verité, quand elle nous presche de fuir la mondaine philosophie, quand elle nous inculque si souvant que nostre sagesse n'est que folie devant Dieu ; que, de toutes les vanitez, la plus vaine c'est l'homme ; que l'homme qui présume de son sçavoir, ne sçait pas encore que c'est que sçavoir ; et que l'homme, qui n'est rien, s'il pense estre quelque chose, se seduit soy mesmes et se trompe ? Ces sentences du sainct esprit expriment si clairement et si vivement ce que je veux maintenir, qu'il ne me faudroit aucune autre preuve contre des gens qui se rendroient avec toute submission et obeïssance à son authorité." $(449)^{50}$

\footnotetext{
49 "Manche behaupten, seine Argumente seien schwach und ungeeignet zu beweisen, was er beweisen will, und sie machen sich stark, sie ohne weiteres umzustoßen. Diese Leute müssen, da atheistisch, etwas härter angepackt werden, denn sie sind gefährlicher und böswilliger als die ersten." (222/1)

${ }^{50}$ "Läßt sich die Stimme der Wahrheit denn überhören, wenn sie uns predigt, die weltliche Philosophie zu fliehen, und wenn sie uns immer wieder einschärft, daß unsere Weisheit vor Gott nur Torheit, daß von allen Eitelkeiten die eitelste der Mensch ist, der sich anmaßt zu wissen und noch nicht einmal weiß, was Wissen heißt, und der, nichts seiend, sich selbst betrügt, wenn er meint, er sei etwas? Dies sind Worte des Heiligen Geistes, und sie drücken auf so klare und lebendige Weise aus, was ich vorzubringen wünsche, daß ich bei Leuten, die sich seiner Autorität in rückhaltlosem Gehorsam unterwürfen, keines weiteren Beweises bedürfte." (222/r)
} 


\section{Die besondere Lage des Menschen als Bürger zweier Welten}

Die Überlegungen Montaignes zu dieser zweiten Argumentation gegen Sebond fallen sehr viel umfangreicher aus, sie beanspruchen fast das ganze Kapitel. Es ist immer wieder bemerkt worden, dass Montaigne in seiner Argumentation zu diesem zweiten Einwand diesen nicht widerlege, sondern vielmehr noch bestärke und stütze. Tatsächlich argumentiert Montaigne mit aller Kraft für die Schwäche derVernunft. Wer den Glauben allein auf die Vernunft glaubt abstützen zu können, ist hochmütig. ${ }^{51}$ Doch dabei wird gerne übersehen, dass es Montaigne um die Zurückweisung einer Position geht, die sich einzig auf die Vernunft stützt. Er leugnet nirgends, dass Gott in seiner Schöpfung deutliche Spuren hinterlasse und er bleibt dabei, dass Glaube auf göttliche Gnade angewiesen ist.

Die Skepsis bezieht sich mehr auf die Details von Sebond, etwa dessen klare aristotelische Seinshierarchie. Aber mit Sebond sieht er den Menschen durch die Gnade mit Gott und durch seinen Körper mit den Tieren verbunden. Montaigne bestreitet den Vorrang des Menschen vor den Tieren, soweit dieser auf natürliche Eigenschaften gründet. Der Mensch erweist sich im Vergleich mit den Tieren auch hinsichtlich seiner Vernunft als keineswegs begünstigt, wie Montaigne mit Bezugnahme auf vornehmlich antike literarische Zeugnisse zu belegen versucht. Zu Sebond liegt hier ein subtiler Unterschied vor: Während dieser, auf Aristoteles und wohl Thomas basierend, eine scala naturae entwirft, welche den Menschen aufgrund seiner Vernunft über die Tiere stellt, sieht Montaigne den Menschen als Naturwesen betrachtet ganz darin eingebettet. ${ }^{52}$ Die Zugehörigkeit zu einem Reich der Gnade hebt den Menschen als Naturwesen nicht über die andern Geschöpfe hinaus.

\footnotetext{
51 "Abbattons ce cuider, premier fondement de la tyrannie du maling esprit: Deus superbis resistit; humilibus autem dat gratiam. L'intelligence est en tous les Dieux, dict Platon, et en fort peu d'hommes." (449) / "Also nieder mit dem Hochmut, diesem Hauptpfeiler der Tyrannei des bösen Geistes! Gott widersteht den Hoffärtigen, doch den Demütigen gewährt er seine Gnade. Vernunft, sagt Platon, ist in allen Göttern, aber in nur ganz wenigen Menschen."(222/1)

52 "J'ay dit tout cecy pour maintenir cette ressemblance qu'il y a aux choses humaines, et pour nous ramener et joindre au nombre. Nous ne sommes ny au dessus, ny au dessoubs du reste: tout ce qui est sous le Ciel, dit le sage, court une loy et fortune pareille." (459) / "All dies sage ich, um die Ähnlichkeit der menschlichen Dinge mit denen der anderen Lebewesen zu betonen und uns in deren große Gemeinschaft zurückzuführen. Wir stehen weder höher noch tiefer als die übrigen Geschöpfe:Alles, was unter dem Himmel ist, sagt der Weise, folgt einerlei Gesetz und Los.« (227/1)
} 
In einem weiteren Schritt untersucht er den Vorteil, den die Vernunft und das Wissen dem Menschen verschafft. Das Resultat ist vernichtend: "J'ay veu en mon temps cent artisans, cent laboureurs, plus sages et plus heureux que des recteurs de l'université, et lesquels j'aimerois mieux ressembler." (487) ${ }^{53}$ Das einfache, gewöhnliche Leben wird von Montaigne in diesem Zusammenhang in vielen Varianten dem Leben der scheinbar Bevorzugten und Edlen nachgerade gepriesen, wohl nicht zuletzt deswegen, weil es die "humaine condition", die zunächst und zumeist einfach "humaine foiblesse» (490) ist, am treffendsten ausdrückt.

"Tant qu'il pensera avoir quelque moyen et quelque force de soy, jamais l'homme ne recognoistra ce qu'il doit à son maistre; il fera tousjours de ses oeufs poules, comme on dit : il le faut mettre en chemise." $(490)^{54}$

Für die Prüfung der Fähigkeiten der menschlichen Vernunft wendet sich Montaigne schliesslich auch der Philosophie zu. In diesem Kontext steht die einzige ausführliche Auseinandersetzung mit der Philosophie in den Essais. Denn die Prüfung der Leistungsfähigkeit der menschlichen Vernunft soll ja nicht an den schwächsten oder auch nur an den gewöhnlichen Vertretern überprüft werden.

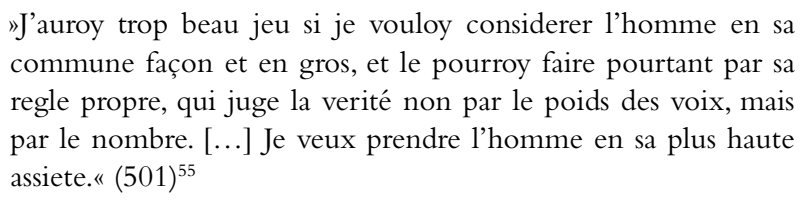

Der Mensch in seiner äussersten Entfaltung, das ist der Philosoph. Doch das dient nur dazu, zu zeigen, dass die Seelenruhe leichter mit dem einfachen Leben des Bauern erreicht wird als durch die Philosophie. Die Philosophie, welche den Anspruch hat, den

53 "Ich habe zu meiner Zeit Hunderte von Handwerkern, Hunderte von Bauern gesehn, die weiser und glücklicher waren als Universitätsrektoren und denen ich daher lieber gleichen möchte." $(242 / \mathrm{r})$

54 "Solang der Mensch glaubt, er verfüge von sich aus über irgendwelche Mittel und Kräfte, wird er niemals anerkennen, was er seinem Herrn zu verdanken hat - nach dem Sprichwort: Das Ei will klüger sein als die Henne. Man muß ihn daher bis aufs Hemd ausziehn." $(243 / \mathrm{r})$

55 "Ich hätte ein leichtes Spiel, wollte ich den Menschen in seiner gewöhnlichen Daseinsweise betrachten, als Massenmensch - seiner eigenen Regel folgend könnte ich es ja ohne weiteres tun, nach der über die Frage, was Wahrheit ist, nicht das Gewicht der Stimme entscheidet, sondern ihre Zahl. [...] Ich will den Menschen vielmehr in seiner äußersten Entfaltung betrachten.«(249/1) 
Menschen am ehesten gut und glücklich zu machen, wird in ihren antiken Ausprägungen zur Zielscheibe der Kritik. Um die Schwäche der Vernunft bei den Philosophen aufzuspüren reicht der Hinweis auf ihre Widersprüche.

"Et le plus large champ aux reprehentions des uns philosophes à l'encontre des autres, se tire des contradictions et diversitez en quoy chacun d'eux se trouve empestré, ou à escient pour montrer la vacillation de l'esprit humain autour de toute matiere, ou forcé ignorammant par la volubilité et incomprehensibilité de toute matiere." $(510)^{56}$

In diesem Zusammenhang äussert Montaigne sein dickes Lob für die Pyrrhoniker, nämlich dass noch nie so Wahrheitsgemässes erfunden worden sei wie eben diese Lehre. Doch beachten wir den ganzen Kontext dieses Lobes:

"Il n'est rien en l'humaine invention où il y ait tant de verisimilitude et d'utilité. Cette-cy presente l'homme nud et vuide, recognoissant sa foiblesse naturelle, propre à recevoir d'en haut quelque force estrangere, desgarni d'humaine science, et d'autant plus apte à loger en soy la divine, aneantissant son jugement pour faire plus de place à la foy; ny mescreant, ny establissant aucun dogme contre les observances communes; humble, obeïssant, disciplinable, studieux; ennemi juré d'hæresie, et s'exemptant par consequant des vaines et irreligieuses opinions introduites par les fauces sectes. C'est une carte blanche preparée à prendre du doigt de Dieu telles formes qu'il luy plaira y graver.« $(506)^{57}$

\footnotetext{
56 „Das weiteste Feld für die wechselseitige Kritik der Philosophen bieten die Ungereimtheiten und Widersprüche, in die jeder von ihnen sich verstrickt - sei es wissentlich, um zu zeigen, wie unstet der menschliche Geist alle Dinge umschwankt, sei es unwissentlich, weil die Dinge zwangsläufig selber schwanken und unbegreiflich bleiben.« (255/1)

57 "Noch nie haben Sterbliche etwas so offensichtlich Wahrheitsgemäßes und Heilsames ersonnen wie die Lehre des Pyrrhon. Sie stellt den Menschen als nackt und leer dar, so daß er seine natürliche Schwachheit erkennt, die ihn für eine von außerhalb, aus der Höhe kommende Kraft empfänglich macht; als allen Menschenwissens entblößt und deshalb zur Aufnahme des göttlichen Wissens um so tauglicher, indem er sein eignes Denken zunichte macht, damit der Glaube mehr Platz in ihm finde; als weder ungläubig noch irgendein Dogma gegen den allgemeinen Brauch aufstellend; als demütig und gehorsam, bildsam und lerneifrig; als geschworenen Feind der Ketzerei und folglich unangefochten von den nichtigen und glaubensfeindlichen Lehren welche durch die dem Irrtum frönenden Schulen eingeführt wurden - als ein weißes Blatt, bereit, den Finger Gottes alle Worte nach dessen Gefallen hierauf niederschreiben zu lassen." $(251 / \mathrm{r})$
} 
Montaigne lobt die Pyrrhoniker dafür, dass sie die Unfähigkeit der menschlichen Vernunft blossgestellt haben, doch seine Weiterung, dass damit dem Glauben Platz gemacht werde, widerspricht dem Geist des Pyrrhonismus. Montaigne nimmt hier fast wörtlich eine spätere Formulierung von Kant vorweg, der davon spricht, die Vernunft zu begrenzen, um dem Glauben Platz zu machen. ${ }^{58}$ Und auch hier haben wir wieder die noch etwas spezifischere Note, dass die Vernunft der Ergänzung durch die göttliche Gnade bedarf.

\section{»Das eigene Sein auf rechte Weise geniessen«}

Hans Stilett hält zu Recht fest, dass sich Montaigne selbst nie als Skeptiker bezeichnet. ${ }^{59}$ Er hebt in seiner Erörterung derVernunft der Philosophen die pyrrhonische Skepsis hervor, er gibt den Fragen den Vorzug vor den Antworten. Das macht ihn gerade so gut zu einem Sokratiker. Und tatsächlich hebt er Sokrates immer wieder lobend hervor. Aber letztlich ist er weder Pyrrho, noch Sokrates, sondern eben Montaigne, der im Frankreich der Religionskriege lebt und sich dezidiert auf die Seite der Tradition stellt, der Sebond verteidigt, und zwar letztlich sehr getreu, indem er mit ihm weniger einen Thomismus, als vielmehr den in seiner Zeit gängigen Augustinismus vertritt: Der schwachen menschlichen Vernunft muss mit der göttlichen Gnade aufgeholfen werden. Montaigne ist damit weniger unser Zeitgenosse, er ist weniger postmodern, als viele zunächst denken. Er vertritt in Glaubensdingen einen ähnlich radikalen Augustinismus wie später der Montaigne-Leser Pascal. Aber er lebt ein ganz anderes Leben als diese beiden. Und zu diesem Leben gehören seine Essais als ständige Erkundung seiner selbst im Spiegel der äusseren Geschehnisse, aber auch und vor allem seiner eigenen Leseerfahrungen.

Auf den letzten Zeilen der Essais bringt Montaigne nochmals zum Ausdruck, um was es ihm so wesentlich zu tun ist: Das Leben eines Menschen, nicht eines Gottes, nicht eines Tieres, das Leben ohne Extravaganzen, das festzuhalten gar nicht so einfach ist. Dass dies das Leben ist, das es anzustreben gilt, das bezweifelt Montaigne nirgends.

"D'autant es tu Dieu comme Tu te recognois homme. C'est une absolue perfection, et comme divine, de scavoyr jouyr loiallement de son estre. Nous cherchons d'autres conditions, pour n'entendre l'usage des nostres, et sortons hors de nous, pour ne sçavoir quel

${ }^{58}$ Vorrede der 2. Auflage der Kritik der reinen Vernunft, BXXX, 1787.

${ }^{59}$ Stilett,Von der Lust, auf dieser Erde zu leben, 2008, 54. 
il y fait. Si avons nous beau monter sur des eschasses, car sur des eschasses encores faut-il marcher de nos jambes. Et au plus eslevé throne du monde si ne sommes assis que sus nostre cul. Les plus belles vies sont, à mon gré, celles qui se rangent au modelle commun et humain, avec ordre, mais sans miracle et sans extravagance. Or la vieillesse a un peu besoin d'estre traictée plus tendrement. Recommandons la à ce Dieu, protecteur de santé et de sagesse, mais gaye et sociale: Frui paratis et valido mihi,/ Latoe, dones, et, precor, integra / Cum mente, nec turpem senectam / Degere, nec cythara carentem." (1115f.) $)^{60}$

Der mit einem Horaz-Zitat ${ }^{61}$ endende Text bringt aber auch nochmals die Dialektik zwischen einfachem Leben und dem Streben nach einem anderen, besseren, zum Ausdruck, welche den Menschen kennzeichnet. Das andere und bessere kann aber nur darin bestehen, das was bereitet ist, durch Einsicht belehrt zu geniessen. Man mag das für heidnisch halten, Montaigne hielt es für christlich. Dass sich im abschliessenden Horaz-Zitat noch ein Bezug auf eine heidnische Gottheit findet, ist für einen akademischen Skeptiker nicht weiter befremdlich: Da wir von Gott keine Kenntnis haben können, sind unsere Bezugnahmen so oder so immer durch unseren beschränkten Blickwinkel bestimmt. Diese Einschätzung teilt er mit Augustinus, seine skeptische Weltsicht, wenn sie denn so bezeichnet werden soll, hat am meisten mit derjenigen des Augustinus gemeinsam. Aber auch hier gilt, dass Montaigne in fast allen andern Dingen einen eigenen Weg geht. Auch wenn die Essais in mancher Hinsicht an die Confessiones erinnern, ist auch hier eine ganz andere Ausrichtung zu erkennen.

- Dr. Alois Rust ist Titularprofessor für Philosophie und Geschäftsleiter der Theologischen Fakultät an der Universität Zürich.

${ }^{60}$ ")Ein Gott wirst du von uns genannt, soweit du dich als Mensch erkannt.< Es ist höchste, fast göttergleiche Vollendung, wenn man das eigene Sein auf rechte Weise zu genießen weiß.Wir suchen andere Lebensformen, weil wir die unsre nicht zu nutzen verstehen; wir wollen über uns hinaus, weil wir nicht erkennen, was in uns ist. Doch wir mögen auf noch zu hohe Stelzen steigen - auch auf ihnen müssen wir mit unsren Beinen gehn; und selbst auf dem höchsten Thron der Welt sitzen wir nur auf unserm Arsch. Meiner Ansicht nach sind jene Leben am schönsten, die sich ins allgemeine Menschenmaß fügen, auf wohlgeordnete Weise, ohne Sonderwünsche, ohne Wundersucht. Das Greisenalter jedoch hat es ein wenig nötig, fürsorglicher behandelt zu werden. Empfehlen wir es in die Hände jenes Gottes, der Gesundheit und Weisheit beschützt - eine fröhliche Weisheit freilich, und gesellige: ‘Lass, o Apoll, was ich erwarb, mich froh genießen, / gesunden Leibes, ungeschwächt an Geist und Seele - / kein schmachvoll Altern mög' des Lebens Lauf beschließen! / Und gib, daß auch der Lyra Labsal mir nicht fehle!« $(566 / \mathrm{r})$

${ }^{61}$ Carmina I, 31. 